\title{
GREGOR observations of a small flare above a sunspot
}

\author{
M. Sobotka ${ }^{1}$, J. Dudík ${ }^{1}$, C. Denker ${ }^{2}$, H. Balthasar ${ }^{2}$, J. Jurčák ${ }^{1}$, \\ W. Liu ${ }^{1}$ and the GREGOR Team \\ ${ }^{1}$ Astronomical Institute of the Czech Academy of Sciences, \\ Fričova 298, 25165 Ondřejov, Czech Republic \\ email: msobotka@asu.cas.cz \\ ${ }^{2}$ Leibniz Institute for Astrophysics Potsdam, \\ An der Sternwarte 16, 14482 potsdam, Germany
}

\begin{abstract}
A small flare ribbon above a sunspot umbra in active region 12205 was observed on November 7, 2014, at 12:00 UT in the blue imaging channel of the 1.5-m GREGOR telescope, using a $0.1 \mathrm{~nm}$ Ca II H interference filter. Context observations from SDO/AIA, Hinode/SOT, and IRIS show that the ribbon is a part of a larger one that extends through the neighboring positive polarities and also participates in several other flares within the active region. A 140 second long time series of Ca II H images was reconstructed by means of the Multi-Frame Blind Deconvolution method, giving the respective spatial and temporal resolutions of 0 ".1 and $1 \mathrm{~s}$. Light curves and horizontal velocities of small-scale bright knots in the observed flare ribbon were measured. Some knots are stationary but three move along the ribbon with speeds of 7 $11 \mathrm{~km} \mathrm{~s}^{-1}$. Two of them move in the opposite direction and exhibit highly correlated intensity changes, providing evidence for the presence of slipping reconnection at small spatial scales.
\end{abstract}

Keywords. Sun: flares, Sun: chromosphere.

\section{Introduction}

Solar flares are explosive phenomena characterized by a strong, rapid increase of electromagnetic radiation throughout the spectrum (e.g., Fletcher et al. 2011). The flare energy originates in the stressed solar magnetic fields and is released by the process of magnetic reconnection (e.g., Priest \& Forbes 2000). Newly reconnected magnetic field lines constitute various observed structures, such as flare loops and the erupting flux rope. In two dimensions, this process is described by the Standard CSHKP solar flare model (see, e.g., Priest 2014) that involves reconnection at a magnetic null-point.

However, in three dimensions, the reconnection can also proceed in the absence of magnetic null-points and the associated topological discontinuities. In these cases, the reconnection occurs in the quasi-separatrix layers (QSLs, Priest \& Démoulin 1995), which are regions where the magnetic connectivity has strong gradients, but is still continuous. The QSLs thus still constitute "boundaries" between different flux systems. They are also associated with local enhancements of the electric current density and correspond well with the observed flare ribbons (Démoulin et al. 1997). The magnetic reconnection in the QSLs is characterized by the apparent slipping motions of the field lines. This occurs because of the continuous exchange of connectivities between reconnecting flux systems (Priest \& Démoulin 1995; Aulanier et al. 2006; Aulanier et al. 2012; Janvier et al. 2013). However, the slippage of the field lines may not correspond to the bulk plasma motions due to the diffusive nature of the reconnection process.

Since QSLs are generalizations of the true topological discontinuities, slipping reconnection occurring within them is a more general mode of magnetic reconnection. Therefore, 

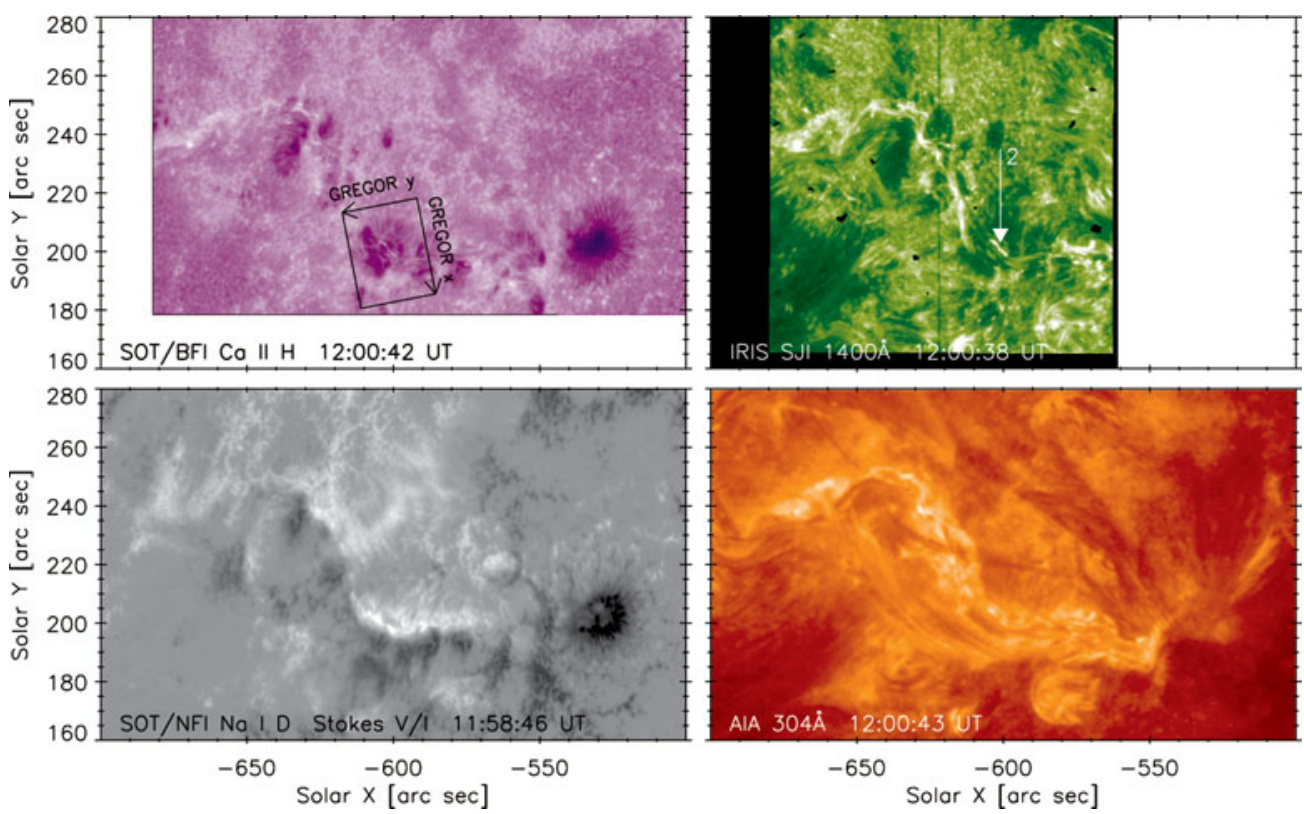

Figure 1. Context observations of AR 12205 from Hinode/SOT (including a magnetogram), IRIS $1400 \AA$ slit-jaw image, and SDO/AIA $304 \AA$ channel. The GREGOR field of view, together with its axes, is shown on the top, left panel. Note that the GREGOR axes are rotated and flipped with respect to the Solar $X$ and $Y$ coordinates. The position of the observed flare ribbon is marked by an arrow in the IRIS image (top, right).

it should be widely observed, especially in solar flares. However, the reports of slipping reconnection were rather scarce until very recently, when they have been found in several solar flares (Dudík et al. 2014, Li \& Zhang 2015). These authors reported individual slipping flare loops or their footpoints observed in UV/EUV filter passbands by the Atmospheric Imaging Assembly (AIA, Lemen et al. 2012; Boerner et al. 2012) onboard the Solar Dynamics Observatory (SDO, Pesnell et al. 2012) or by the Interface Region Imaging Spectrograph spacecraft (IRIS, De Pontieu et al. 2014).

In this paper, we report on optical imaging observations with ultra-high spatial and temporal resolution performed in the blue imaging channel (BIC, Puschmann et al. 2012) in the line core of Ca II H $396.8 \mathrm{~nm}$ with the new 1.5-meter GREGOR telescope (Schmidt et al. 2012) at the Observatorio del Teide, Tenerife, Spain.

\section{Context observations}

The Active Region NOAA 12205 (hereafter, AR 12205) was located at position 13N, $37 \mathrm{E}$ on November 7,2014 . This active region had a $\beta \gamma \delta-\beta \gamma \delta$ magnetic configuration and was the site of many flares, including six C-class ones, two M-class ones, and one X1.6-class flare during November 7 alone. The active region, as observed at multiple wavelengths by different space-borne instruments is displayed in Fig. 1. This figure shows the active region at approximately 12:00 UT, corresponding to the time of the observations performed by the GREGOR telescope, which acquired images of a small flare ribbon within a sunspot umbra during the period 11:59:08-12:01:31 UT (see Sect. 3).

Shortly after the GREGOR observations, the GOES 1-8 A X-ray flux started rising at 12:04 UT and peaked at 12:06 UT during a C3.9 flare. This means that we very probably detected a pre-flare phase in our GREGOR Ca II H data. The site of the C3.9 flare was 

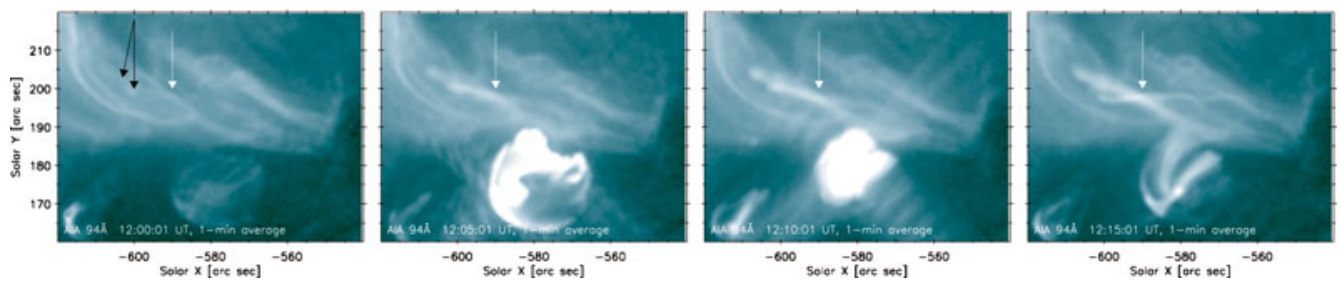

Figure 2. Evolution of the AIA $94 \AA$ emission after the GREGOR observations. From the left, $t=12: 00,12: 05,12: 10$, and 12:15 UT. The black arrows point to the location of the ribbon observed by GREGOR. The white arrow shows the Fe XVIII loops whose footpoints lie in the GREGOR ribbon. The circular ribbon at the bottom shows a strong brightening at 12:05 and 12:10 UT during the C3.9 flare

a circular ribbon seen in the AIA $304 \AA$ channel (see Fig. 1 bottom, right and Fig. 2). Its location as well as several transient brightenings preceding the flare were visible already around 12:00 UT. These transient brightenings occured mostly along a long, ribbon-like structure apparent in the Hinode/Solar Optical Telescope (SOT, Tsuneta et al. 2008) Ca II H observations, in the IRIS $1400 \AA$ slit-jaw images, or in the SDO/AIA $304 \AA$ channel. This ribbon-like structure corresponded to the footpoints of hot Fe XVIII loops observed in the AIA $94 \AA$ band (Fig. 2). Together with the portion of the ribbon within the $\delta$-spot observed by GREGOR and the circular ribbon are all lying in the positive polarities. The conjugate footpoints of the flare loops, according to AIA $94 \AA$, are located in the negative polarities in the vicinity of the leading sunspot.

\section{GREGOR observations}

The GREGOR observations of the pre-flare phase (hereafter called a "flare" for simplicity) were made during the "Early Science" period of the telescope, using the integrated adaptive optics system (Berkefeld et al. 2012). The field of $35 " \times 27 "$, including the flare and underlying sunspot, was observed in the blue imaging channel equipped with a $0.1 \mathrm{~nm}$ Ca II H interference filter centered at $396.8 \mathrm{~nm}$. A series of 12-bit frames was acquired with the frame rate $7.677 \mathrm{~Hz}$, exposure time $30 \mathrm{~ms}$, and image scale 0.026 arcsec per pixel from 11:55:13 to 12:01:31 UT. After the standard dark- and flat-field corrections, some residual fringes specific for the pointing position of the telescope remained in the field of view. To remove them, the frames at the beginning of the series (11:55:13-11:59:07 UT), i.e., before the flare appeared, were averaged and the rest of the series with the flare was divided by this average. In addition to the fringes, this operation removed also real structures present before the onset of the flare, including the sunspot. The flare ribbon was not influenced and the resulting series of flare images (11:59:08-12:01:31 UT) had intensities normalized to those before the flare beginning.

The Multi-Frame Blind Deconvolution method (MFBD, van Noort et al. 2005) was then applied to this series. Sequences of 32 frames were used to obtain one restored image and the restoration was made in a sliding mode, with a step of 8 frames (frames 1-32 produced the first restored image, frames 9-40 the second, etc.), so that 132 restored images with a time step of $1.04 \mathrm{~s}$ were obtained. Taking into account the smallest resolved features, the resulting spatial resolution was 0".1.

Small bright knots are clearly resolved in the flare ribbon. The Local Correlation Tracking algorithm (LCT, November \& Simon 1988) with a tracking window of 0".5 was applied to the whole restored series to estimate the dynamics of the ribbon (Fig. 3, left). In addition to the expansion of the ribbon area, motions with speeds of $5-12 \mathrm{~km} \mathrm{~s}^{-1}$ 

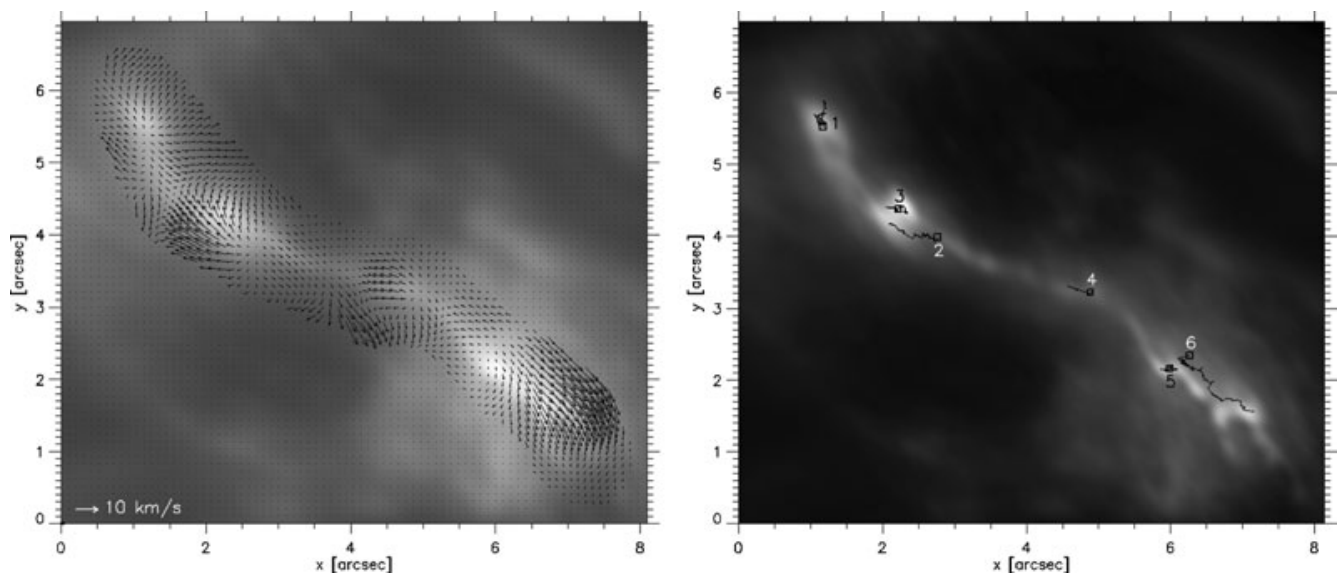

Figure 3. Tracking of motions in the flare ribbon. Left: LCT flow map with underlying average image of the series. Right: Trajectories of knots 1-6 with the best image in the background. Small squares denote starting positions of the tracking.
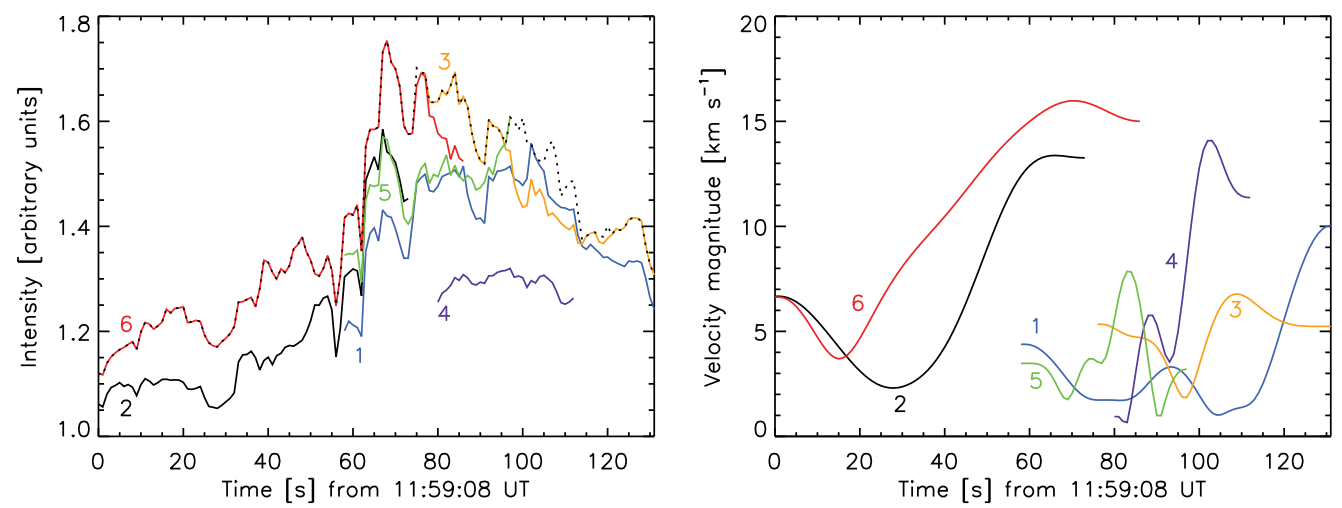

Figure 4. Intensities and horizontal velocities of bright knots 1-6. Left: Light curves. The intensities are in units of the initial intensity before the flare. The black dotted line represents the absolute intensity maximum in the ribbon. Right: Low-pass filtered instantaneous velocity magnitudes. Knot 6 moves in a direction opposite to the directions of knots 2 and 4 .

in both directions along the ribbon are detected in three places. More information can be obtained by tracking individual bright knots. The procedure locks on the tracked feature using the spatial correlation of intensities between two neighboring frames in a $1 " .24 \times 1 " .24$ window around the feature and records the value and $(x, y)$ position of its intensity maximum in each frame. Six bright knots (1-6) were tracked successfully and their trajectories are shown in the right panel of Fig. 3. Due to the effects of splitting and merging that interrupted the tracking, they were not followed during their whole lifetime. The tracking periods range from $32 \mathrm{~s}$ (knot 4) to $86 \mathrm{~s}$ (knot 6$)$.

Resulting light curves and velocity profiles of the tracked knots are shown in Fig. 4. The intensities are in units of the initial intensity before the flare. The knots 2 and 6 , tracked from the beginning of the series, first show a slow increase of brightness, then a sudden brightening ( $t=63 \mathrm{~s}$ after the beginning, seen also in knots 1 and 5$)$, an intensity maximum (knot $6, I=1.75, t=68 \mathrm{~s}$ ) and a decrease. The linear correlation coefficient for the intensity evolution of knots 2 and 6 during their common $73 \mathrm{~s}$ tracking time is high, 0.97, suggesting a common process as the origin of both knots. Three knots $(1,3,5)$ follow 
a similar intensity evolution, but their positions oscillate randomly around the initial positions. Knots 2 and 4 show a translatory motion along the ribbon in one direction, while the knot 6 moves in the opposite. Instantaneous velocities of the knots calculated from their positions are very noisy (a shift of 1 pixel represents $18.75 \mathrm{~km} \mathrm{~s}^{-1}$ ). Applying a low-pass Fourier filter, the error was reduced to $\pm 4 \mathrm{~km} \mathrm{~s}^{-1}$. The resulting time-dependent velocity magnitudes are plotted in the right panel of Fig. 4, showing acceleration phases of $0.2-0.7 \mathrm{~km} \mathrm{~s}^{-2}$ typical for the moving knots 2,4 , and 6 . These knots have average velocities (and standard deviations) $7 \pm 1,8 \pm 2$, and $11 \pm 2 \mathrm{~km} \mathrm{~s}^{-1}$, respectively. The opposite motion of the knots 2 and 6 , together with their highly correlated intensity evolution, indicates the presence of slipping reconnection.

\section{Discussion and conclusions}

Based on the behavior of knots 2 and 6, i.e., the oppositely-oriented velocities along the ribbon, the similar behavior of the respective velocities, and the highly correlated intensity evolution, we propose that these two knots are footpoint locations of two flare loops undergoing slipping reconnection. In principle, the slipping reconnection (Aulanier et al. 2006) and the change of connectivity of the corresponding loops should be visible in EUV observations.

However, due to an order-of-magnitude difference between the GREGOR and AIA pixel size (0".026 vs. 0".6) and spatial resolution (0".1 vs. 1".5), we are not able to resolve the corresponding individual, thin flare loops in the AIA datasets. Nevertheless, the inspection of AIA images shows that post-reconnection loops anchored in the ribbon observed by GREGOR are indeed present and are getting brighter (Fig. 2). These loops are prominent in AIA $94 \AA$, meaning that the temperature is sufficient for formation of Fe XVIII. To confirm this, we also inspected the AIA $131 \AA$ and $171 \AA$ images (not shown). The loop emission is present but faint in $131 \AA$ and not visible in $171 \AA$, suggesting that they are indeed Fe XVIII loops with a faint Fe XXI component, i.e., flare loops; and not coronal loops emitting in Fe VIII-X instead.

The evolution of the AIA $94 \AA$ emission shows that the reconnection-heated loops indeed change their footpoints. At 12:15 UT, two crossing loops anchored in the ribbon observed previously by GREGOR are clearly visible. The crossing point is denoted by the white arrow in Fig. 2, while the location of the GREGOR ribbon is denoted by a pair of black arrows in the 12:00 UT image. These observations suggest that slipping reconnection may be continuing for about 10-15 minutes after the end of the GREGOR observations.

We presented GREGOR observations of slipping bright knots in a small ribbon-like structure within a sunspot umbra. Multi-wavelength context observations were used to establish the relation of this ribbon to other flaring activity within AR 12205. The fact that the GREGOR observations precede the C3.9 flare suggests that the slipping reconnection is occurring also outside of major flaring events. The high spatial resolution of GREGOR shows that the slipping reconnection can occur on small spatial scales that are unresolvable by instruments such as AIA (cf. Dudík et al. 2014). These observations illustrate the strong need for sub-arcsecond spatial resolution in order to elucidate the dynamics and the energy release in the solar atmosphere.

\section{Acknowledgements}

This work was supported by the grants 14-04338S (MS, JJ), P209/12/1652 (JD) of the Czech Science Foundation, and DE 7873.1 (CD) of the Deutsche Forschungsgemeinschaft. 
WL was supported by the European Comission's FP-7 Collaborative Project No. 606862 "F-CHROMA". The support of the FP-7 Capacities Project No. 312495 "SOLARNET" and the institutional support RVO:67985815 of the Academy of Sciences of the Czech Republic is also acknowledged. The 1.5-meter GREGOR solar telescope was built by a German consortium under the leadership of the Kiepenheuer Institute for Solar Physics in Freiburg with the Leibniz Institute for Astrophysics Potsdam, the Institute of Astrophysics Göttingen, and the Max Planck Institute for Solar System Research in Göttingen as partners, and with contributions by the Instituto de Astrofísica de Canarias and the Astronomical Institute of the Czech Academy of Sciences. The SDO/AIA data are available by courtesy of NASA/SDO and the AIA science team. Hinode is a Japanese mission developed and launched by ISAS/JAXA, with NAOJ as domestic partner and NASA and STFC (UK) as international partners. It is operated by these agencies in co-operation with ESA and NSC (Norway). IRIS is a NASA small explorer mission developed and operated by LMSAL with mission operations executed at NASA Ames Research Center and major contributions to downlink funded by NSC, Norway, through an ESA PRODEX contract.

\section{References}

Aulanier, G., Janvier, M., \& Schmieder, B. 2012, Astron. Astrophys., 543, A110

Aulanier, G., Pariat, E., Démoulin, P., \& DeVore, C. R. 2006, Solar Phys., 238, 347

Berkefeld, T., Schmidt, D., \& Soltau, D., et al. 2012, Astronomische Nachrichten, 333, 863

Boerner, P., Edwards, C., \& Lemen, J., et al. 2012, Solar Phys., 275, 41

De Pontieu, B., Title, A. M., \& Lemen, J. R., et al. 2014, Solar Phys., 289, 2733

Démoulin, P., Bagala, L. G., \& Mandrini, C. H., et al. 1997, Astron. Astrophys., 325, 305

Dudík, J., Janvier, M., Aulanier, G., et al. 2014, Astrophys. J., 784, 144

Fletcher, L., Dennis, B. R., \& Hudson, H. S., et al. 2011, Space Sci. Rev., 159, 19

Janvier, M., Aulanier, G., Pariat, E., \& Démoulin, P. 2013, Astron. Astrophys., 555, A77

Lemen, J. R., Title, A. M., \& Akin, D. J., et al. 2012, Solar Phys., 275, 17

Li, T. \& Zhang, J. 2015, Astrophys. J., 804, L8

November, L. J. \& Simon, G. W. 1988, Astrophys. J., 333, 427

Pesnell, W. D., Thompson, B. J., \& Chamberlin, P. C. 2012, Solar Phys., 275, 3

Priest, E. 2014, Magnetohydrodynamics of the Sun (Cambridge: Cambridge University Press)

Priest, E. \& Forbes, T. 2000, Magnetic Reconnection (Cambridge: Cambridge University Press)

Priest, E. R. \& Démoulin, P. 1995, J. Geophys. Res., 1002, 23443

Puschmann, K. G., Denker, C., \& Kneer, F., et al. 2012, Astronomische Nachrichten, 333, 880

Schmidt, W., von der Lühe, O., Volkmer, R., et al. 2012, Astronomische Nachrichten, 333, 796

van Noort, M., Rouppe van der Voort, L., \& Löfdahl, M. G. 2005, Solar Phys., 228, 191

Tsuneta, S., Ichimoto, K., \& Katsukawa, Y., et al. 2008, Solar Phys., 249, 167 